\title{
Chemical studies of some fused heterocyclic compounds containing oxygen with anticipated biological interests
}

\author{
Aya A. El-Sawi, Mohamed Abozeid, El-Sayed I. El-Desoky and Abdelrahman H. Abdelrahman
} Department of Chemistry, Faculty of Science, Mansoura University, Egypt

\section{III}

Background: Coumarin compounds are one of the most active classes of heterocyclic compounds. They play active roles in medicine due to their biological activity. These compounds are found in many natural products. Aim: This study aimed to synthesize some new compounds containing oxygen and nitrogen. The newly prepared compounds are evaluated biologically as antituberculosis, antiinflammatory, and anti-tumors. Also, some computational measurement studies were carried out as docking, drug prediction, and modeling. Materials and Methods: The present work divided into three parts: Part 1: Unorthodox synthesis, biological activity, and DFT studies of novel and multi functionalized naphthoxocine derivatives Part 2: Synthesis of some novel heterocyclic compounds derived from naphthopyranone-3-carboxaldehyde and 3-carbonitrile. Results: The first pathway concern with the effect of mononucleophiles such as 4-amino antipyrine and 2-amino benzothiazole. The second pathway promotes the effect of 1,2-N, N-nucleophiles such as hydrazine hydrate. The third pathway study the effect of 1,3-C, N-nucleophiles such as cyanoacetamide and $\mathrm{N}$-(aryl or hetero aryl) derivatives to building up the nicotinonitrile derivatives. The fourth direction the reactivity towards a variety of 1,4-N, N-(or N, S-) binucleophiles. Part 3: Computational and biological evaluation the newly prepared compounds are evaluated pharmaceutically as antituberculosis, anti-inflammatory, and antitumors. Also, computational measurement studies were carried out as docking, drug prediction, and modeling. Conclusion: The studies fused heterocyclic compounds containing oxygen showed appreciated biological activities.

Keywords: Antituberculosis; DFT studies; Naphthoxocine 